\title{
Flutuação populacional e comparação de métodos de coleta de Aedes (Stegomyia) aegypti (Diptera, Culicidae)
}

\author{
Eduardo B. Beserra, Paulino S. Ribeiro \& Sueide A. de Oliveira
}

Departamento de Biologia, Universidade Estadual da Paraíba, Rua Juvêncio Arruda, s/n, Campus Universitário, Bodocongó, 58109-790 Campina Grande, PB, Brasil. (ebarbosa@uepb.edu.br; paulsribeiro@yahoo.com.br; saos_amaral@hotmail.com)

\begin{abstract}
Floating population and comparison of collection method of Aedes (Stegomyia) aegypti (Diptera, Culicidae). Environmental factors act as regulators of expanding populations of Aedes aegypti (Linnaeus, 1762). This study aimed to evaluate the influence of temperature, relative air humidity, and pluviometric precipitation on the population dynamics of Ae. aegypti. The research was conducted at Boqueirão, Campina Grande and Remígio, state of Paraíba, comprising an 12-month period, using the method of collects per trap oviposition and larvae research. The population fluctuation of eggs and other immature forms as well as the infestation rates for eggs and larvae were correlated to climatic data using the Pearson correlation analysis. The chi-square and Tukey tests were used to compare the infestation between the study areas, and efficiency between research methods with trap oviposition and larval collecting. No correlations were found between climate variables and population dynamics of Ae. aegypti, neither with the indexes for oviposition trap (IAO) and Breteau (IB). The IAO was considered high, with an average of 54.9, 59.53 and $59.51 \%$, while the IB was very low with an average of 1.55, 1.69 and 3.64, for the municipalities of Boqueirão, Campina Grande and Remígio, respectively. The chi-square analysis for the number of positive properties was highly significant, and we may infer that the collection method for oviposition trap was more efficient than the larvae research in the detection of infestation by Ae. aegypti. The results indicated that the evaluated factors cannot be considered isolated on ecological analysis of the distribution of this species, since correlations were not found between vector presence and climatic factors such as temperature, relative air humidity and rainfall. So in the ecological analysis of the distribution of Ae. aegypti should be considered the multiple constraints and determinants of its infestation.
\end{abstract}

KEYWORDS. Vector, ecology, distribution, collection methodology.

RESUMO. Fatores ambientais podem atuar como reguladores da expansão das populações de Aedes aegypti (Linnaeus, 1762). Este trabalho objetivou avaliar a influência da temperatura, umidade relativa do ar, e precipitação pluviométrica na flutuação populacional de Ae. aegypti. A pesquisa foi conduzida em Boqueirão, Campina Grande e Remígio, Estado da Paraíba, compreendendo um período de 12 meses, utilizando-se o método de coleta por armadilha de oviposição e de pesquisa larvária. A flutuação populacional de ovos e de outras formas imaturas, bem como os índices de infestação para ovos e larvas foi correlacionada com os dados climáticos utilizando-se a análise de correlação de Pearson. Os testes do qui-quadrado e de Tukey foram utilizados para comparar a infestação entre as áreas de estudo e a eficiência entre os métodos de pesquisas com armadilha de oviposição e de coletas de larvas. Não foram encontradas correlações entre as variáveis climáticas e a flutuação populacional de $A e$. aegypti, nem com os índices para armadilha de oviposição (IAO) e de Breteau (IB). O IAO foi considerado alto, com média de 54,9; 59,53 e 59,51\%, enquanto que o IB foi muito baixo com média de 1,55, 1,69 e 3,64, para os municípios de Boqueirão, Campina Grande e Remígio, respectivamente. A análise do qui-quadrado para o número de imóveis positivos foi altamente significativa, podendo-se inferir que o método de coleta por armadilha de oviposição foi mais eficiente que a pesquisa larvária na detecção da infestação pelo Ae. aegypti. Os resultados indicam que os fatores avaliados não podem ser considerados isolados na análise ecológica da distribuição desta espécie, já que não foram estabelecidas correlações entre a presença do vetor e fatores climático, como temperatura, umidade relativa do ar e precipitação pluviométrica. Assim na análise ecológica da distribuição do Ae. aegypti deve ser considerados os múltiplos fatores condicionantes e determinantes da sua infestação.

PALAVRAS-CHAVE. Vetor, ecologia, distribuição, metodologia de coleta.

Espécies de Aedes são pragas em áreas urbanas e rurais amplamente distribuídas em todo o mundo. Larvas de muitas espécies vivem em água potável. Aedes aegypti (Linnaeus, 1762) deposita seus ovos em potes e frascos de armazenamento de água localizados dentro ou fora das casas. Essa espécie geralmente não ocorre em áreas onde as temperaturas noturnas são abaixo de $20^{\circ} \mathrm{C}$ (RoBinson, 2005).

Aedes aegypti vem demonstrando características de rápida adaptação ecológica a ambientes urbanos cada vez mais complexos, distribuindo-se por todas as áreas urbanas e impondo cada vez mais dificuldades para o estabelecimento dos programas de controle baseados no seu monitoramento. Essas dificuldades têm estimulado novos estudos de biologia, comportamento e a relação desta espécie com o espaço urbano. Resultados mais recentes têm contribuído para uma revisão sobre parâmetros comportamentais de Ae. aegypti, em particular, aqueles sobre deslocamento e dispersão em áreas urbanas, taxa de sobrevivência, estratégias de oviposição, caracterização de áreas de vida, entre outros (REITER \& GUBLER, 1997; REITER et al., 2003; Reiter, 2007; Beserra \& CASTro JR, 2008; Regis et al., 2010; CASTRO JR. et al., 2013; GAMBARRA et al., 2013).

O Brasil concentra mais de $80 \%$ da população na área urbana, com importantes lacunas no setor de infraestrutura, tais como dificuldades para garantir o abastecimento regular e contínuo de água, a coleta e o destino adequado dos resíduos sólidos (BRASIL, 2009). Outros fatores, como a acelerada expansão da indústria de materiais não biodegradáveis, além de condições climáticas favoráveis, agravadas pelo aquecimento global, conduzem a um cenário que impede, em curto prazo, a proposição de ações visando à erradicação do vetor. 
O conhecimento da influência da sazonalidade sobre a capacidade de dispersão, reprodução e adaptação de $A e$. aegypti aos ambientes urbanos é fundamental, pois implica em um melhor gerenciamento de estratégias de controle do vetor e maior eficiência para redução do número de casos de dengue. Desta forma, torna-se necessário o desenvolvimento de estudos bioecológicos partindo-se da premissa de que populações de Ae. aegypti de regiões diferentes apresentam distinção quanto às variáveis biológicas como o período de desenvolvimento, capacidade reprodutiva e capacidade de dispersão de fêmeas.

Este trabalho teve como objetivo confrontar os métodos de coleta para captura de Ae. aegypti, comparando o potencial de produção de adultos de criadouros peri e intradomiciliares em função do seu volume e potabilidade, bem como estimar a influência de fatores abióticos sobre a flutuação populacional em áreas urbanas da Paraíba.

\section{MATERIAL E MÉTODOS}

Flutuação das populações de Ae. aegypti mediante a utilização de armadilha para oviposição e de pesquisa larvária. A pesquisa ocorreu nas cidades de Boqueirão, Campina Grande e Remígio, Paraíba (PB), entre dezembro de 2005 a dezembro de 2006, perfazendo um período de 12 meses de coleta. Em cada município selecionou-se dois bairros com condições socioeconômicas diferenciadas, levando-se em consideração o número de imóveis, as condições de infraestrutura (higiênico-sanitária), como a canalização interna de água, canalização interna de esgoto, coleta de lixo e pavimentação de ruas, e os índices de infestação do Ae. aegypti e de dengue, fornecidos pelas Secretarias Municipais de Saúde, e Secretaria Estadual de Saúde. Foram considerados bairros com melhores condições de infraestrutura aqueles que apresentam acima de $80 \%$ de canalização interna de água, de canalização interna de esgoto, de coleta de lixo e de pavimentação de ruas. Com relação à infestação por Ae. Aegypti, foi estimado a partir dos índices de infestação predial, escolhendo-se aqueles bairros com índices próximos ou acima de 5\% durante o $3^{\circ}$ ciclo de 2004.

Em cada bairro selecionou-se dez quarteirões sendo consideradas aleatoriamente as residências para realização de coletas. Nos bairros Prata e Nova Brasília, em Campina Grande, sorteou-se 50 residências, e nos bairros Centro e Palmas em Remígio, e Bairro Novo e Bela Vista em Boqueirão, foram selecionados aleatoriamente, 30 residências. Em cada residência, a cada 30 dias, instalouse uma armadilha para a coleta de ovos (ovitrampa). A armadilha consiste de um balde plástico de coloração preta (capacidade 2L) contendo 1 litro de água; no interior das mesmas utilizou-se como substrato de oviposição, palhetas de eucatex com dimensão de 2,5 cm de largura x $12,0 \mathrm{~cm}$ de comprimento, presas por um clipe à sua parede interna.

As armadilhas eram recolhidas a cada quatro dias após a instalação e o material coletado, acondicionado em caixas térmicas de isopor e levados ao laboratório para posterior quantificação do número de ovos. As palhetas com os ovos eram depositadas em bandejas plásticas contendo água desclorada através de filtro desclorador acoplado à torneira de rede de água do Laboratório de Entomologia e, quando atingiam o quarto estágio $\left(\mathrm{L}_{4}\right)$, as larvas foram montadas em lâminas e sob microscópio realizada a identificação da espécie.

A obtenção das variáveis meteorológicas de cada município, como temperatura, umidade relativa do ar e precipitação pluviométrica se deu através do Laboratório de Meteorologia, Recursos Hídricos e Sensoriamento Remoto da Paraíba, onde correlacionou-se à flutuação populacional de Ae. aegypti.

Dados referentes ao número de ovos foram tabulados utilizando-se o programa Excel/Windows para posterior processamento e análise. Determinou-se o índice de infestação para armadilha de oviposição (IAO) pela fórmula: $\mathrm{IAO}=\left(\mathrm{n}^{\mathrm{o}}\right.$ de casas com armadilhas positivas $/ \mathrm{n}^{\circ}$ de casas inspecionadas) x 100

Avaliou-se a densidade populacional de formas imaturas em cada localidade mediante amostras mensais, no intra e peridomicílio. A coleta de larvas e pupas se deu em todos os depósitos existentes nos domicílios selecionados. A inspeção era realizada por meio de concha entomológica, constituída de uma concha metálica com capacidade de $500 \mathrm{ml}$, percorrendo-se a superfície da água e em seguida todo o volume da água até o fundo, com movimentos em zigue-zague, coletando-se larvas e pupas ali existentes. Não havendo profundidade suficiente para a utilização do pesca larva, realizou-se inspeção visual para a contagem. Acondicionaram-se os espécimes coletados em sacos plásticos, etiquetados, e levados ao Laboratório de Entomologia da Universidade Estadual da Paraíba, para contagem e identificação.

A tabulação dos dados referentes ao número de larvas por área ocorreu utilizando-se o programa Excel/Windows para processamento e análise sendo, posteriormente determinados: (1) Índice de Breteau (IB), geral para cada localidade e específico para tipo de depósito segundo categorização por volume e potabilidade, a saber: IB = $\left(\mathrm{n}^{\mathrm{o}}\right.$ de recipientes positivos $/ \mathrm{n}^{\circ}$ de domicílios examinados) x 100; (2) Análise dos dados e comparação dos métodos de pesquisa com armadilha de oviposição e de pesquisa larvária: a flutuação populacional de ovos e outras formas imaturas, bem como os índices de infestação para ovos e larvas foram correlacionadas com os dados de temperatura, umidade relativa e precipitação pluviométrica das áreas de estudo, utilizando-se a análise de correlação de Pearson.

Para comparar a infestação entre as áreas de estudo e a eficiência entre os métodos de pesquisas com armadilha de oviposição e de coletas de larvas utilizou-se o teste de Quiquadrado para independência e o teste de comparação de média de Tukey. Para o teste de Tukey, os dados analisados consistiam em esquema fatorial $3 \mathrm{X} 2$, considerando os três municípios (Boqueirão, Campina Grande e Remígio) e dois bairros em cada município. 


\section{RESULTADOS}

Correlação entre a flutuação populacional de Aedes aegypti e variáveis climáticas. A análise de correlação de Pearson entre o número de ovos/coleta mês, o Índice para armadilha de oviposição (IAO) e as variáveis climáticas de cada região não foram significativas (Tab. I) nas localidades estudadas.

Devido ao reduzido número de formas imaturas (larvas e pupas), optou-se por analisar os dados referentes à pesquisa larvária considerando-se a soma mensal de indivíduos nos dois bairros de cada município. Como constatado para o número de ovos/coleta mês e o IAO, a análise de Pearson, não mostrou correlações significativas entre o número de indivíduos imaturos, o Índice de Breteau (IB) e as variáveis climáticas para os municípios de Boqueirão, Campina Grande e Remígo (Tab. II).

Como o número de indivíduos imaturos coletados durante a pesquisa larvária foi baixo, insuficientes para a comparação da infestação entre as áreas de estudo, optouse por utilizar apenas os dados referentes à coleta com armadilha para oviposição, como parâmetro de comparação dessas áreas. A análise de variância não detectou interação significativa entre municípios e bairros, bem como não demonstrou diferença significativa com relação aos IAOs dos municípios de Boqueirão, Campina Grande e Remígio (Fig. 1). Porém a análise do qui-quadrado para o número de imóveis positivos $\left(\mathrm{X}^{2}=11,4 ;\right.$ g.l. $\left.=2,0 ; \mathrm{P}<0,01\right)$ foi altamente

Tab. I. Correlação entre o número de ovos e o índice para armadilha de oviposição (IAO) de Aedes aegypti (Linnaeus, 1762) e variáveis climáticas dos municípios de Boqueirão, Campina Grande e Remígio, Paraíba, Brasil.

\begin{tabular}{|c|c|c|c|c|c|c|}
\hline & \multicolumn{3}{|c|}{ Número de ovos } & \multicolumn{3}{|c|}{$\mathrm{IAO}$} \\
\hline & $\mathrm{t}$ & $\mathrm{df}$ & $\mathrm{p}$ & $\mathrm{T}$ & df & $\mathrm{p}$ \\
\hline & \multicolumn{6}{|c|}{ Boqueirão } \\
\hline & \multicolumn{6}{|c|}{ Bairro Novo } \\
\hline Temperatura & $-0,7705$ & 10 & 0,4588 & $-1,2168$ & 10 & 0,2516 \\
\hline Umidade relativa do ar & $-0,9764$ & 10 & 0,3519 & $-0,8374$ & 10 & 0,4219 \\
\hline \multirow[t]{2}{*}{ Precipitação } & $-1,1587$ & 10 & 0,2735 & $-0,5698$ & 10 & 0,5814 \\
\hline & \multicolumn{6}{|c|}{ Bela Vista } \\
\hline Temperatura & $-1,0282$ & 10 & 0,3281 & $-2,1278$ & 10 & 0,0592 \\
\hline Umidade relativa do ar & $-0,1745$ & 10 & 0,8650 & $-0,383$ & 10 & 0,7097 \\
\hline \multirow[t]{3}{*}{ Precipitação } & $-0,5021$ & 10 & 0,6264 & $-0,115$ & 10 & 0,9107 \\
\hline & \multicolumn{6}{|c|}{ Campina Grande } \\
\hline & \multicolumn{6}{|c|}{ Prata } \\
\hline Temperatura & $-2,008$ & 10 & 0,075 & $-2,576$ & 10 & 0,029 \\
\hline Umidade relativa do ar & 2,200 & 10 & 0,055 & 0,857 & 10 & 0,413 \\
\hline \multirow[t]{2}{*}{ Precipitação } & 1,5198 & 10 & 0,158 & 0,4921 & 10 & 0,6333 \\
\hline & \multicolumn{6}{|c|}{ Nova Brasília } \\
\hline Temperatura & $-0,282$ & 10 & 0,783 & $-0,355$ & 10 & 0,730 \\
\hline Umidade relativa do ar & 2,862 & 10 & 0,018 & 1,506 & 10 & 0,116 \\
\hline \multirow[t]{3}{*}{ Precipitação } & 1,9223 & 10 & 0,0834 & 1,7203 & 10 & 0,1161 \\
\hline & \multicolumn{6}{|c|}{ Remígio } \\
\hline & \multicolumn{6}{|c|}{ Centro } \\
\hline Temperatura & $-0,578$ & 10 & 0,575 & $-0,965$ & 10 & 0,358 \\
\hline Umidade relativa do ar & 0,745 & 10 & 0,472 & 0,953 & 10 & 0,362 \\
\hline \multirow[t]{2}{*}{ Precipitação } & 0,6671 & 10 & 0,5198 & 0,4117 & 10 & 0,6893 \\
\hline & \multicolumn{6}{|c|}{ Palmas } \\
\hline Temperatura & $-0,051$ & 10 & 0,960 & $-0,750$ & 10 & 0,470 \\
\hline Umidade relaiva do ar & $-0,725$ & 10 & 0,485 & 1,192 & 10 & 0,260 \\
\hline Precipitação & $-1,1096$ & 10 & 0,2931 & $-0,6147$ & 10 & 0,5525 \\
\hline
\end{tabular}

Tab. II. Correlação entre o número de indivíduos imaturos de Aedes aegypti (Linnaeus, 1762) e o Índice de Breteau (IB) e as variáveis climáticas dos municípios de Boqueirão, Campina Grande e Remígio, Paraíba, Brasil.

\begin{tabular}{|c|c|c|c|c|c|c|}
\hline & \multicolumn{3}{|c|}{ Número de imaturos } & \multicolumn{3}{|c|}{ IB } \\
\hline & $\mathrm{t}$ & $\mathrm{df}$ & $\mathrm{p}$ & $\mathrm{T}$ & df & $\mathrm{P}$ \\
\hline & \multicolumn{6}{|c|}{ Boqueirão } \\
\hline Temperatura & 1,0098 & 8 & 0,3422 & 1,0488 & 8 & 0,3249 \\
\hline Umidade relativa do ar & $-0,1629$ & 8 & 0,8752 & $-0,19$ & 7 & 0,9854 \\
\hline \multirow[t]{2}{*}{ Precipitação } & $-0,8476$ & 8 & 0,4213 & 0,8402 & 8 & 4252 \\
\hline & \multicolumn{6}{|c|}{ Campina Grande } \\
\hline Temperatura & 1,1979 & 7 & 0,2699 & 1,4184 & 7 & 0,1990 \\
\hline Umidade relativado ar & $-0,7329$ & 7 & 0,4874 & $-1,189$ & 7 & 0,2732 \\
\hline \multirow[t]{2}{*}{ Precipitação } & $-0,8827$ & 7 & 0,4067 & $-1,0544$ & 7 & 0,3267 \\
\hline & \multicolumn{6}{|c|}{ Remígio } \\
\hline Temperatura & 2,9674 & 9 & 0,0157 & 2,467 & 9 & 0,0357 \\
\hline Umidade relativa do ar & 0,5509 & 9 & 0,5951 & 0,3466 & 9 & 0,7369 \\
\hline Precipitação & 0,8007 & 9 & 0,4439 & 0,621 & 9 & 0,55 \\
\hline
\end{tabular}




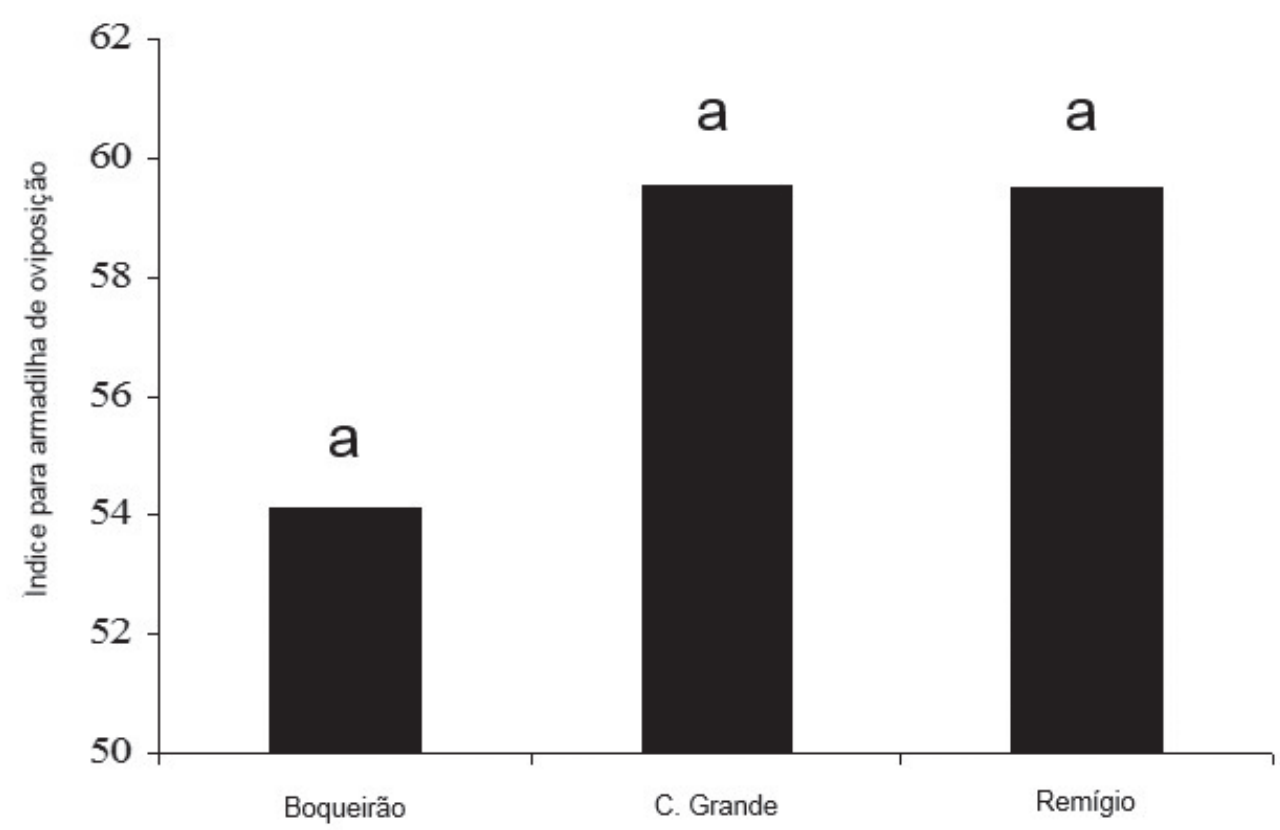

Fig.1. Índice para armadilha de oviposição (IAO) (\%) dos municípios de Boqueirão, Campina Grande e Remígio, Paraíba, Brasil. Histogramas seguidos de mesmas letras não diferem entre si pelo teste $\mathrm{F}(\mathrm{P}<0,05)$.

significativa, podendo-se concluir que Campina Grande apresentou maior infestação que os demais municípios (Fig. 2). Apesar dessa maior infestação para o município de Campina Grande, pode-se considerar que em todos os municípios os IAOs estavam elevados durante todos os meses de coletas (Tab. III), com média de 54,9; 59,53 e $59,51 \%$, respectivamente para os municípios de Boqueirão, Campina Grande e Remígio.

Comparando-se os bairros em cada município não houve diferença no número de imóveis positivos entre o bairro Novo e Bela Vista em Boqueirão ( $\mathrm{X}^{2}=1,8$; g.l. = 1,0; $\mathrm{P}<0,05)$. Em Campina Grande, Nova Brasília mostrou-se com maior infestação que o bairro da Prata $\left(\mathrm{X}^{2}=6,11\right.$; g.l. $=1,0 ; \mathrm{P}<0,05)$, enquanto que em Remígio, o centro da cidade foi a área de maior infestação se comparado ao bairro Palmas $\left(\mathrm{X}^{2}=7,83\right.$; g.1.= 1,0; $\left.\mathrm{P}<0,01\right)$ (Figs 3-5).

Comparação entre os métodos de coleta por armadilha para oviposição e de pesquisa larvária. $O$ método de coleta de armadilha de oviposição obteve um número médio de ovos por coleta/mês de 777,6 e 680,2 na Prata e Nova Brasília, 339,6 e 307,6,0 no bairro Novo e Bela Vista, e de 478,1 e 419,5 em Palmas e Centro de Remígio, respectivamente. Os IAOs se mantiveram altos nos meses de coleta. Os menores IAOs foram para bairro Novo e Bela Vista em Boqueirão, e Prata em Campina Grande em dezembro de 2005 (IAO $=8 \%, 6,7 \%$ e $10 \%$,

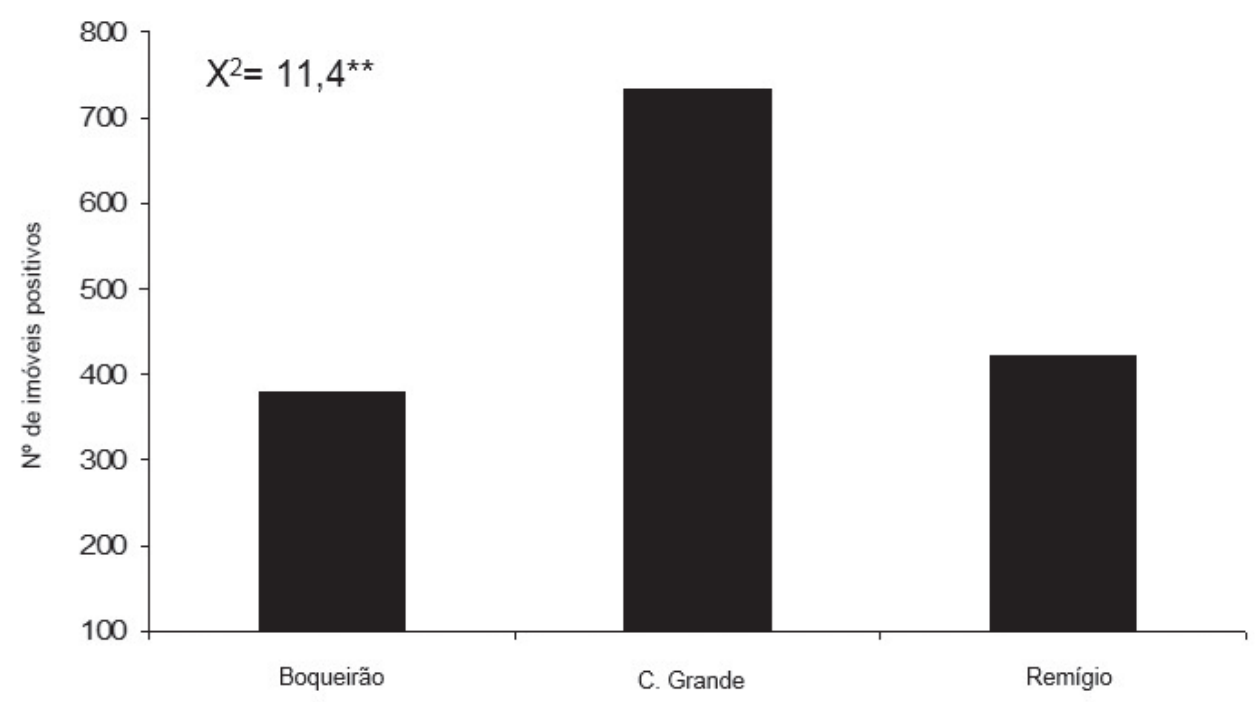

Fig. 2. Histogramas do número de imóveis positivos para Aedes aegypti (Linnaeus, 1762) nos municípios de Boqueirão, Campina Grande e Remígio, Paraíba, Brasil (**, qui-quadrado significativo a $1 \%$ de probabilidade). 
Tab. III. Índice de infestação para armadilha de oviposição (IAO) a partir de coletas mensais de populações de Aedes aegypti (Linnaeus, 1762), no município de Boqueirão, Campina Grande e Remígio, Paraíba, Brasil $\left[{ }^{1}, \mathrm{IAO}=\left(\mathrm{n}^{\circ}\right.\right.$ de domicílios com armadilhas positivas/total de domicílios inspecionados) $\mathrm{x}$ 100].

\begin{tabular}{|c|c|c|c|c|c|c|}
\hline \multirow{3}{*}{ Período de coleta } & \multicolumn{6}{|c|}{ IAO/Localidades ${ }^{1}$} \\
\hline & \multicolumn{2}{|c|}{ Boqueirão } & \multicolumn{2}{|c|}{ Campina Grande } & \multicolumn{2}{|c|}{ Remígio } \\
\hline & Bairro Novo & Bela Vista & Prata & Nova Brasília & Centro & Palmas \\
\hline $12 / 2005$ & 8 & 6,7 & 11,8 & 42,55 & 20,7 & 26,7 \\
\hline $01 / 2006$ & 10 & 30 & 29,2 & 16 & 43,3 & 53,3 \\
\hline $02 / 2006$ & 16,7 & 13,3 & 66 & 31 & 40 & 73,3 \\
\hline $03 / 2006$ & 23,3 & 40 & 45,6 & 32 & 46,3 & 53,3 \\
\hline $04 / 2006$ & 20 & 36,7 & 48,9 & 46 & 86,7 & 46,7 \\
\hline $05 / 2006$ & 83,3 & 60 & 76 & 40 & 90 & 50 \\
\hline $06 / 2006$ & 65,5 & 63,3 & 68 & 35 & 43,3 & 66,7 \\
\hline $07 / 2006$ & 54,5 & 53,3 & 82 & 32 & 83,3 & 66,7 \\
\hline $08 / 2006$ & 82,7 & 66,6 & 78 & 28 & 66,7 & 53,3 \\
\hline $09 / 2006$ & 76 & 76,7 & 95,8 & 41 & 80 & 50 \\
\hline $10 / 2006$ & 93,3 & 66,7 & 38 & 38 & 40,1 & 53,3 \\
\hline $11 / 2006$ & 76,7 & 53,3 & 68 & 25 & 88,9 & 26,7 \\
\hline $12 / 2006$ & 80 & 56,7 & - & - & 60 & 60 \\
\hline
\end{tabular}

respectivamente) e bairro Novo (Boqueirão) em janeiro de $2006(\mathrm{IAO}=10 \%)$; em todos os demais períodos de coletas os índices foram acima de $20 \%$, chegando-se a obter IAOs superiores a $90 \%$ no bairro Novo em outubro de 2006, e na Prata em setembro de 2006 (Tab. III). A pesquisa larvária, através da utilização de pesca larva, coletou em média/mês 1,$88 ; 3,9$ e 5,5 imaturos (larvas e pupas) de Ae. aegypti, com IB médio de 1,55; 1,69 e 3,64 para os municípios de Boqueirão, Campina Grande e Remígio, respectivamente (Tab. IV). A análise do qui-quadrado para o número de imóveis positivos $\left(\mathrm{X}^{2}=1563,1\right.$; g.l. $=1,0$; $\mathrm{P}<0,01)$ foi altamente significativa, podendo-se concluir que o método de coleta por armadilha de oviposição foi mais eficiente que o da pesquisa larvária na detecção de infestação por Ae. aegypti (Fig. 6).

\section{DISCUSSÃO}

No estudo não foram estabelecidas correlações entre a presença de Ae. aegypti e as condições climáticas das regiões estudadas. Dessa forma, a temperatura, a umidade e a precipitação não influenciaram na flutuação populacional de ovos, larva e pupas, e nos IAOs e IBs. Do fato de não ocorrer correlação entre essas variáveis, pode se inferir que durante todo ano há condições ótimas, principalmente de temperatura, para o estabelecimento deste inseto, como constatado por BESERRA et al. (2006) para os municípios de Boqueirão, Campina Grande e Remígio, e que fatores outros, como os epidemiológicos humanos, podem influenciar no estabelecimento e ocorrência de focos do vetor, independente das condições climáticas de cada região. De acordo com Coelho (2012), além de fatores climáticos, a falta de infraestrutura das cidades propicia uma situação permanente de vulnerabilidade, o que leva principalmente a oferecer alto potencial de recipientes artificiais para reprodução do mosquito.

Silva et al. (2003) verificaram em Umuarama, no Paraná, que os meses de janeiro e fevereiro, período de alta densidade pluviométrica, são os mais favoráveis à infestação do Ae. aegypti e a ocorrência de surtos de dengue, o que é corroborado pelos dados de Souza-Santos (1999), que encontrou forte correlação entre a ocorrência de formas imaturas desse vetor e fatores meteorológicos, como temperatura, umidade e precipitação, no estado do

Tab. IV. Índice de Breteau (IB) a partir de coletas mensais de larvas e pupas de Aedes aegypti (Linnaeus, 1762), nos município de Boqueirão, Campina Grande e Remígio, Paraíba, Brasil [1, IAO= ( $\mathrm{n}^{\mathrm{o}}$ de domicílios com armadilhas positivas/total de domicílios inspecionados $\left.) \mathrm{x} 100\right]$.

\begin{tabular}{cccc}
\hline \multirow{2}{*}{ Período de coleta } & & IAO/Localidades ${ }^{1}$ & \\
\cline { 2 - 4 } & Boqueirão & Campina Grande & 20,33 \\
\hline $12 / 2005$ & 7,27 & 4,0 & 6,66 \\
$01 / 2006$ & 5,0 & 3,77 & 3,33 \\
$02 / 2006$ & 1,66 & 6,12 & 3,38 \\
$03 / 2006$ & 1,66 & 0,0 & 5,0 \\
$04 / 2006$ & - & 1,04 & 3,33 \\
$05 / 2006$ & 0,0 & 0,0 & 1,66 \\
$06 / 2006$ & 0,0 & 0,0 & 0,0 \\
$07 / 2006$ & - & 10,0 & 0,0 \\
$08 / 2006$ & 0,0 & 0,0 & - \\
$09 / 2006$ & - & - & 0,0 \\
$10 / 2006$ & 0,0 & - & 0,0 \\
$11 / 2006$ & 0,0 & - & 0,0 \\
$12 / 2006$ & 0,0 & 1,69 & 3,64 \\
Média & 1,55 & & \\
\hline
\end{tabular}



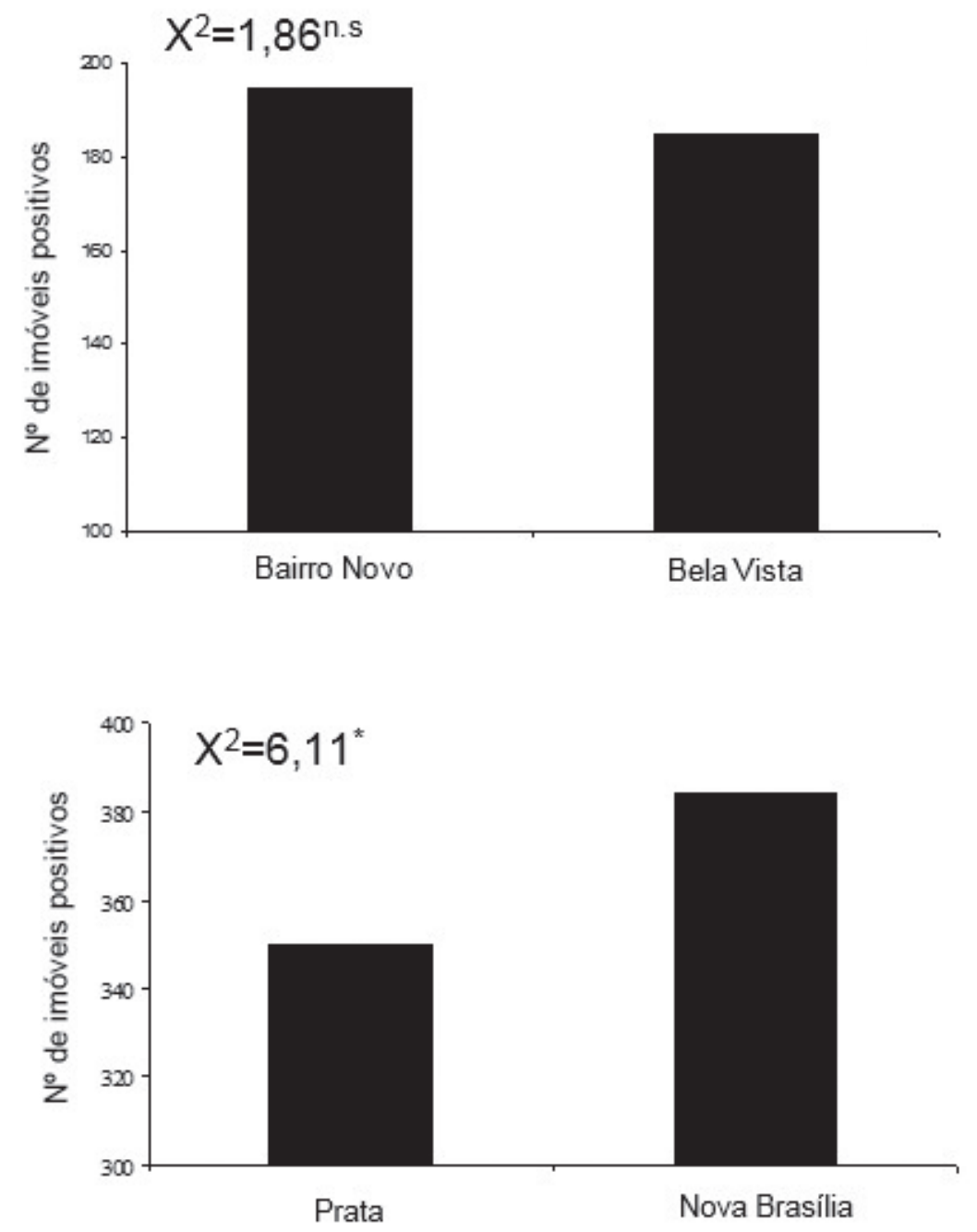

4

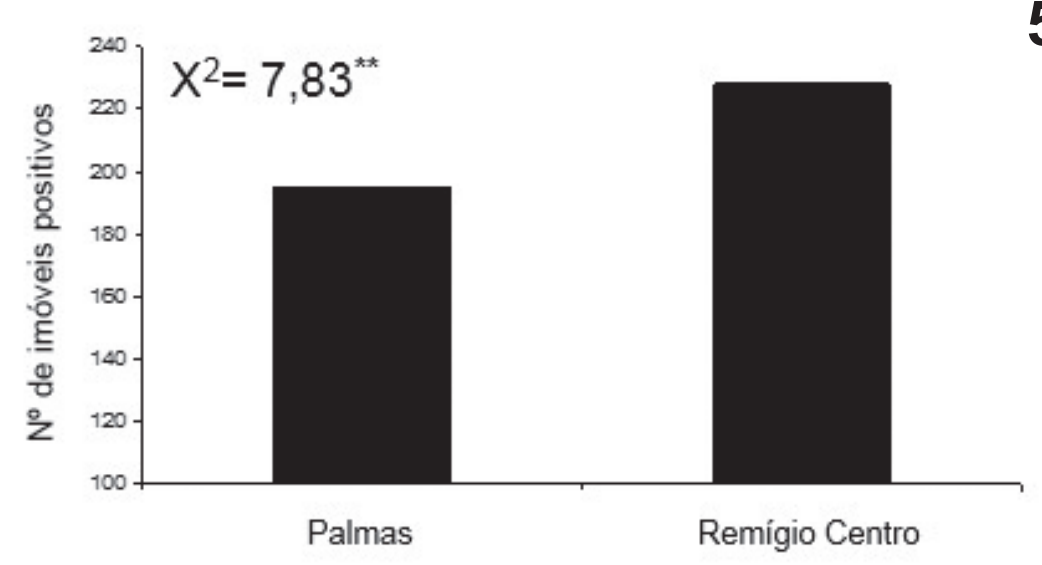

Figs 3-5. Histogramas do número de imóveis positivos para Aedes aegypti (Linnaeus, 1762) nos bairros dos municípios: 3, Boqueirão; 4, Campina Grande e 5, Remígio, Paraíba, Brasil ( ${ }^{\text {n.s. }}$. não significativo; *, ** significativo a 5 e $1 \%$ respectivamente, pelo teste de qui-quadrado ). 


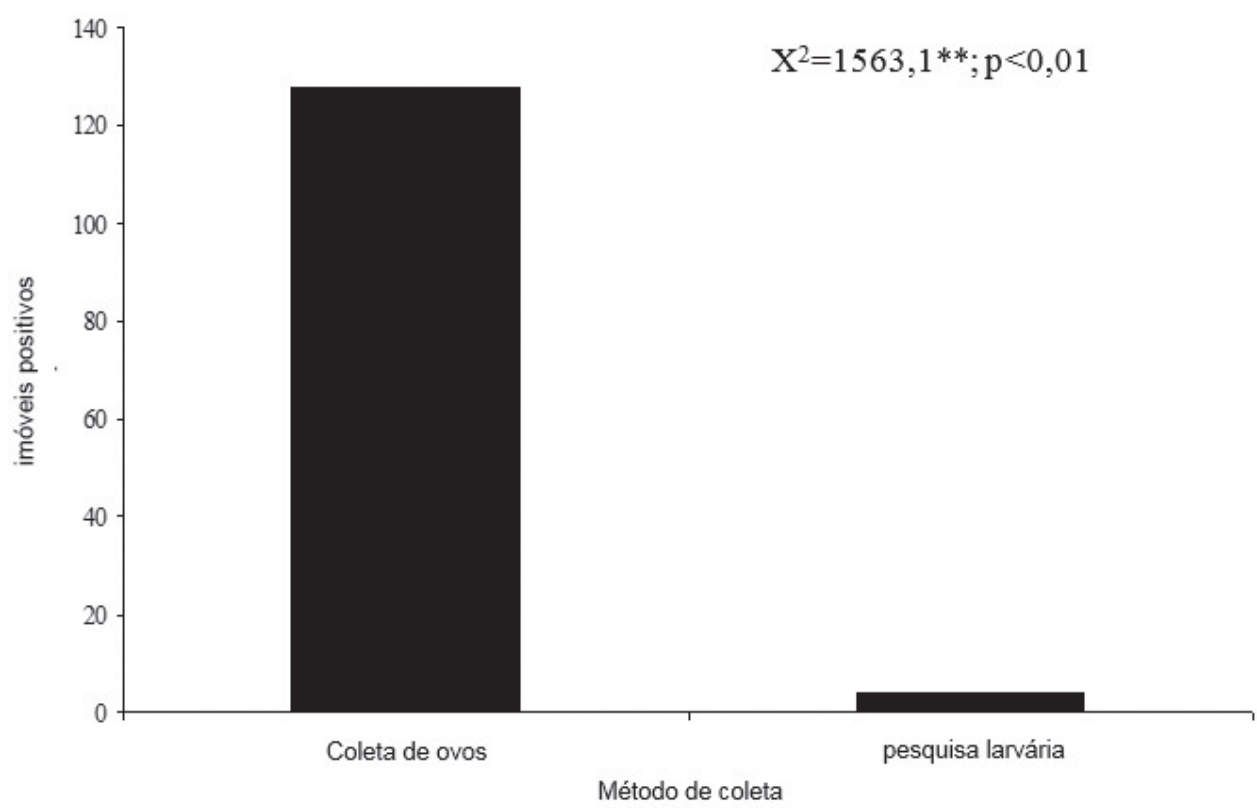

Fig. 6. Média do número de imóveis positivos/mês detectados através do método de coleta de armadilha de oviposição e da pesquisa larvária através de pesca larva (**, qui-quadrado significativo a $1 \%$ de probabilidade).

Rio de Janeiro, e de SteIN et al. (2005) que registraram maior quantidade de ovos de $A$. aegypti nos meses de novembro e dezembro, período de maiores precipitação e temperatura na Província do Chaco na Argentina.

MiYAZAKI et al. (2009) no Mato Grosso e em Cuiabá verificou que as temperaturas máxima, média e mínima do ar, associadas a precipitações pluviométricas foram significativas quando associadas ao número de ovos, mas não foram detectadas correlações entre o número de ovos e umidade relativa do ar. Esses autores citaram que outras causas contribuíram de modo mais preponderante para os resultados encontrados.

Fatores humanos que podem ter interferido nos resultados desta pesquisa são de natureza operacionais, como o controle vetorial. A aplicação de inseticida durante o período de coleta pode diminuir a população do vetor, independente da estação do ano e, consequentemente, o número de indivíduos coletados, exercendo maior influência na dinâmica populacional de Ae. aegypti do que os fatores climáticos investigados. Além disso, deve-se também levar em consideração o papel da população humana, tanto no controle, quanto na criação e manutenção de possíveis criadouros, que favorecem a população do vetor (SouzASantos \& CARvalho, 2000).

Vale ressaltar que os fatores epidemiológicos e operacionais humanos não foram objetos de estudo no presente projeto, mas, necessitam ser melhores investigados, para que se possa desenvolver um modelo de previsão quanto ao aumento populacional de Ae. aegypti.

A análise da infestação das áreas mostrou que todos os locais de coleta são áreas potenciais de risco de surtos de dengue, já que apresentaram altos índices de incidência do vetor com IAOs médios de 54,9; 59,53 e 59,51\% e IBs de 1,55, 1,69 e 3,64 para os municípios de Boqueirão, Campina Grande e Remígio. Embora os IBs, possam ser considerados baixos como indicadores da presença de $A e$. aegypti, o mesmo não pode ser considerado quando se avalia o risco de incidência da dengue, já que, segundo a Organización Pan-Americana de la Salud (1995) existem relatos de epidemia de dengue em locais com índice de infestação predial de $1 \%$.

De acordo com TexeIRA et al. (1999), epidemias de dengue já foram observadas em locais com índice de infestação predial da ordem de $1 \%$ a $2 \%$. Vale ressaltar que, apesar dos IBs terem sido baixos, ocorreram altos IAOs, que dá uma indicação maior da presença do inseto adulto.

A pesquisa larvária mostrou-se pouco eficiente em detectar a ocorrência de imóveis positivos, mesmo em locais onde os métodos de armadilha para oviposição acusaram altos índices de infestação. O Índice de Breteau (IB) manteve-se baixo em todos os períodos de coleta e em todos os municípios, com exceção do município de Remígio que em dezembro de 2005, obteve-se um IB de 20,33\% (Tab. IV). Os resultados são próximos aos observados por SERPA et al. (2006) que, ao analisarem a sazonalidade de Ae. aegypti e Ae. albopictus no município de Potim, estado de São Paulo, demonstraram frequência abundante de Ae. aegypti em todas as estações do ano e na totalidade de pneus pesquisados. Outras contribuições como a de Calderón-Arguedas et al. (2004) em San José na Costa Rica, obtiveram IBs que variaram de 3,06 a 9,09. Por outro lado, o método de coleta por armadilha de oviposição, apesar de mais trabalhoso por requer um maior número de armadilhas e tempo, mostrou-se extremamente eficiente na detecção de Ae. aegypti, evidenciando sua presença durante todos os meses de coleta (Tab. III). 
A comparação entre os métodos de coleta sugere a importância da utilização de um parâmetro seguro, que indique com maior precisão a densidade populacional de indivíduos em determinada localidade. O método de armadilha para oviposição é seguro para a deteç̧ão da presença da fêmea do Ae. aegypti e pode ser utilizado em estudos dessa natureza.

Os resultados sugerem ainda que os fatores avaliados não podem ser considerados isolados na análise ecológica da distribuição desta espécie, já que não foram estabelecidas correlações entre estes e a presença do vetor. Assim, na análise ecológica da distribuição do Ae. Aegypti, devem ser considerados os múltiplos fatores condicionantes e determinantes da sua infestação.

Agradecimentos. À Secretaria Estadual de Saúde da Paraíba e a Secretaria de Saúde dos municípios de Boqueirão, Campina Grande e Remígio; ao $3^{\circ}$ Núcleo Regional de Saúde; à Fundação Nacional de Saúde (FUNASA), pelo apoio durante as instalações das armadilhas; ao Laboratório de Recursos Hídricos e Sensoriamento Remoto, pela disponibilização de dados climáticos; à Organização Pan Americana de Saúde (OPAS/OMS), pelo financiamento através da carta acordo (BR/ LOA/0500008.002 e BR/LOA/0700026.001); ao PIBIC/UEPB/CNPq, pela bolsa de iniciação científica.

\section{REFERÊNCIAS BIBLIOGRÁFICAS}

Beserra, E. B. \& Castro-Júnior, F. P. 2008. Biologia Comparada de Populações de Aedes (Stegomyia) aegypti (L.) (Diptera: Culicidae) da Paraíba. Neotropical Entomology 37(1):81- 85.

Beserra, E. B.; Castro-Júnior, F. P.; dos Santos, J. W.; Santos, T. S. \& Fernandes, C. R. M. 2006. Biologia e exigências térmicas de Aedes aegypti (L.) (Diptera: Culicidae) provenientes de quatro regiões bioclimáticas da Paraíba. Neotropical Entomology 35(6):853-860.

BRASIL. 2009. Diretrizes nacionais para prevenção e controle de epidemias de dengue. Brasília, Ministério da Saúde, Secretaria de Vigilância em Saúde. 160p.

Calderón-Arguedas, O.; Troyo, A. \& Solano, M. E. 2004. Caracterizacón de los sitios de multiplicación de Aedes aegypti (Diptera: Culicidae) en el casério "La Carpio", San José, Costa Rica durante la estación seca del año 2003. Revista Biomédica15(2):73-79.

Castro-Júnior, F. P.; Martins, W. F.; Lucena Filho, M. L.; Almeida, R. P. \& BeserRa, E. B. 2013. Ciclos de vida comparados de Aedes aegypti (Diptera: Culicidae) do semiárido da Paraíba. Iheringia, Série Zoologia 103(2):118-123.

Coelno, G. 2012. Challenges in the control of Aedes aegypti. Revista do Instituto de Medicina Tropical de São Paulo 54:13-14.

Gambarra, W. P. T.; Martins, W. F. S.; Lucena Filho, M. L.; Albuquerque, I. M. C.; Apolinário, O. K. S. \& Beserra, E. B. 2013. Spatial distribution and esterase activity in populations of Aedes (Stegomyia) aegypti (Linnaeus) (Diptera: Culicidae) resistant to temephos. Revista da Sociedade Brasileira de Medicina Tropical 46(2):176-184.
Miyazaki, R. D.; Ribeiro, A. L. M.; Pignatti, M. G., Campelo Júnior, J. H. \& Pignati, M. 2009. Monitoramento do mosquito Aedes aegypti (Linnaeus, 1762) (Diptera: Culicidae), por meio de ovitrampas no Campus da Universidade Federal de Mato Grosso, Cuiabá, Estado de Mato Grosso. Revista da Sociedade Brasileira de Medicina Tropical 42(4):392-397.

Organización Pan-Americana de la Salud. 1995. Dengue y dengue hemorrágico en las Américas: guias para su prevencion y control. Washington D.C., OPS- Publicacion Científica.

Regis, L.; Acioli, R. V.; Walter, C. M. N.; Serafim, J.; Silveira JR, J. C.; Souza, W. V.; Ribeiro Jr, P. J.; Melo-Santos, M. A. V. \& Monteiro, A. M. 2010. Dados recentes sobre impacto da remoção mecânica de ovos e adultos sobre densidades populacionais de Aedes aegypti em dois municípios pernambucanos. In: IV Workshop de Genética e Biologia molecular de Insetos Vetores de Doenças Tropicais, 2010, Recife. Resumos do ENTOMOL-IV4:31-34.

ReITER, P. 2007. Oviposition, dispersal, and survival in Aedes aegypti: implications for the efficacy of control strategies. Vector-Borne Zoonotic Diseases 7:261-274.

Reiter, P. \& Gubler, D. J. 1997 Surveillance and control of urban dengue vectors. In: Gubler, D. J. \& Kuno, G. eds. Dengue and Dengue Hemorrhagic Fever. New York, CAB International, p. 425-462.

Reiter, P.; Lathrop, S.; Bunnig, M.; Biggerstaff, B.; Singer, D.; Tiwari, T.; Baber, L.; Amador, M.; Thirion, J.; Hayes, J.; SeCA, C.; Mendez, J.; Ramirez, B.; Robinson, J.; Rawlings, J.; Vorndam, V.; Waterman, S.; Gubler, D.; Clark, G. \& Hayes, E. 2003. Texas Lifestyle Limits Transmission of Dengue Virus. Emerging Infectious Diseases 9(1):86-89.

Robinson, W. H. 2005. Urban insects and arachnids - A Handbook of Urban Entomology. New York, Cambridge University Press. 480p.

Serpa, L. L. N.; Costa, K. V. R. M.; Voltolini, J. C. \& Kakitani, I. 2006. Variação sazonal de Aedes aegypti e Aedes albopictus no município de Potim, São Paulo. Revista de Saúde Pública 40(6):1-5.

Silva, A. A.; Miranda, C. F.; Ferreira, J. R. \& Araújo, E. J. de A. 2003. Fatores sociais e ambientais que podem ter contribuído para a proliferação da dengue em Umuarama, estado do Paraná. Acta Scientiarum 25(1):81-85.

SouZA-SAntos, R. 1999. Fatores associados à ocorrência de formas imaturas de Aedes aegypti na Ilha do Governador, Rio de Janeiro, Brasil. Revista da Sociedade Brasileira de Medicina Tropical 32(4):373-382.

Souza-Santos, R. \& Carvalho, M. S. 2000. Análise da distribuição espacial de larvas de Aedes aegypti na Ilha do Governador, Rio de Janeiro, Brasil. Cadernos de Saúde Pública 16(1):31-42.

Stein, M.; Oria, G. I.; Almirón, W. R. \& Willener, J. A. 2005. Fluctuación estacional de Aedes aegypti en Chaco, Argentina. Revista de Saúde Pública 39(4):559-564.

Teixeira, M. G.; Barreto, M. L. \& Guerra, Z. 1999. Epidemiologia e medidas de prevenção do dengue. Informe epidemiológico do SUS8(4):5-33. 